\title{
ASYMPTOTICAL WELL BEHAVIOUR FOR CONSTRAINED MINIMISATION PROBLEMS
}

\author{
D. Aussel and C.C. Chou
}

\begin{abstract}
This paper is devoted to the study of the links between stationary sequence and minimising sequence for constrained minimisation problems. The constraint set is not supposed to be convex and no differentiability assumption is made on the objective function. New tools are developed in this general framework and we prove a necessary and a sufficient condition for such problems to have a "constrained asymptotical well behaviour" (that is, each stationary sequence is a minimising sequence). Our work extend that of Auslender, Cominetti and Crouzeix.
\end{abstract}

\section{INTRODUCTION}

Let us consider the general constrained optimisation problem

$$
m=\inf _{c \in C} f(c)
$$

where $f$ is a lower semicontinuous function from a Banach space $X$ to $\mathbb{R} \cup\{+\infty\}$ and $C$ is a nonempty subset of $X$. Numerical methods, being in general based on descent methods, generate stationary sequences (that is, in the differentiable unconstrained case, sequences such that $\nabla f\left(x_{n}\right)$ tends to 0 whenever $\left.n \rightarrow+\infty\right)$. It is thus crucial to be able to answer to the following question (already raised by Powell [22] in 1976): for which classes of optimisation problems one can ensure that each stationary sequence is a minimising sequence $\left(f\left(x_{n}\right) \rightarrow m\right.$ whenever $\left.n \rightarrow+\infty\right)$ ?

Besides the simple case of the minimisation in a finite dimensional space under some compactness assumptions on $C$ or on $f$ (inf-compactness), a first answer has been given in Auslender and Crouzeix [2] and Auslender, Cominetti and Crouzeix [3]. Here the authors obtained a characterisation of nonsmooth unconstrained convex problems (of proper closed convex functions) having an asymptotical well behaviour, that is, each stationary sequence (that is, the distance of $\partial f\left(x_{n}\right)$ to 0 tends to 0 ) is a minimising sequence.

Some extensions to nonconvex unconstrained problems have been proposed in Penot [19] and Huang, $\mathrm{Ng}$ and Penot [15], while a dual characterisation and the study of the 
multiobjective case are respectively due to Angleraud [1] and Bernoussi, Bolintineanu and Chou [6]. The concept of asymptotical well behaviour is strongly linked to the notion of well-posedness (see $[17,18,19,20]$ and references therein).

For constrained problems, the only attempt, to our knowledge, is a paper of Chou, $\mathrm{Ng}$ and Pang [12] in which they consider problems with continuously differentiable objective functions and convex constraint set. This lack of interest in constrained well behaviour is essentially due to the fact that constrained problems are often considered as particular cases of unconstrained problems by simply adding the indicator function of $C$ to the objective function (see for example, [15]). But this reduction of the problem is not adapted to the algorithmic point of view, and does not allow us to consider asymptotically feasible sequences $\left(d\left(x_{n}, C\right)\right.$ tends to 0$)$. Thanks to our approach we shall be able to work with such sequences (see Definition 3.2 and Theorem 4.4).

Our aim in this paper is to develop a new theory of asymptotical well behaviour adapted to constrained optimisation. In Section 2 we define the fundamental concept of the local infimum value, and Section 3 is devoted to the study of basic properties of stationary sequences and minimising sequences in the setting of constrained optimisation problems. Finally in Section 4 we prove our main result, namely, a sufficient condition (Theorem 4.4) for a nonconvex constrained optimisation problem to have an constrained asymptotical well behaviour. This result, unlike those of [12], is established in infinite dimensional space and does not need any differentiability assumption on the function nor any convexity hypothesis on the constraint set.

\section{Local infimum VALUE}

Throughout this paper, $X$ stands for a real Banach space, $X^{*}$ for its topological dual and $\langle\cdot, \cdot\rangle$ for the duality pairing. For $\varepsilon>0,(x, y) \in X^{2}$ and $\|\cdot\|$ a norm on $X$, we use the notations

$$
B_{\varepsilon}(x)=\{y \in X:\|y-x\|<\varepsilon\}
$$

and

$$
\bar{B}_{\varepsilon}(x)=\{y \in X:\|y-x\| \leqslant \varepsilon\} .
$$

For any subset $C$ of $X$, $\operatorname{int}(C), \bar{C}$ (or $\operatorname{cl}(C)$ ), $d_{C}(x)$ or $d(x, C), B_{\varepsilon}(C)$ will denote respectively the interior of $C$, the closure of $C$, the distance $d_{C}(x)=d(x, C)=\inf _{c \in C}\|x-c\|$ and the open subset $B_{\varepsilon}(C)=\left\{x \in X: d_{C}(x)<\varepsilon\right\}$.

Finally for a lower semicontinuous function $f: X \rightarrow \mathbb{R} \cup\{+\infty\}$, dom $f$ $=\{x \in X: f(x)<+\infty\}$, if $\lambda \in \mathbb{R}, L(\lambda)$ is the $\lambda$-sublevel set of $f$ (that is $L(\lambda)=\{x \in X: f(x) \leqslant \lambda\}$ ), and $\partial f$ is the Clarke-Rockafellar subdifferential of $f$ defined by

$$
\partial f(x):=\left\{x^{*} \in X^{*} \mid\left\langle x^{*}, v\right\rangle \leqslant f^{\dagger}(x, v), \quad \forall v \in X\right\}
$$


with

$$
f^{\uparrow}(x, v)=\sup _{\varepsilon>0} \limsup _{\substack{x^{\prime} \rightarrow f^{x} \\ t \searrow^{x}}} \inf _{v^{\prime} \in B_{\varepsilon}(v)} \frac{f\left(x^{\prime}+t v^{\prime}\right)-f\left(x^{\prime}\right)}{t} .
$$

Let us recall that the Clarke-Rockafellar subdifferential of $f$ coincides with the Fenchel subdifferential

$$
\partial^{F e n} f(x)=\left\{x^{*} \in X^{*}:\left\langle x^{*}, y-x\right\rangle \leqslant f(y)-f(x), \forall y \in X\right\}
$$

whenever the function $f$ is supposed to be convex. It is also well known that $\partial f$ coincides with the classical Clarke subdifferential ([9]) if $f$ is locally Lipschitz.

Now let $f: X \rightarrow \mathbb{R} \cup\{+\infty\}$ be a lower semicontinuous function and $C$ be a nonempty subset of $X$. Since no convexity assumption will be made on $C$, a natural way to envisage the minimisation of $f$ on $C$ is to turn our attention to local infimum concepts. But, on the other hand, since $f$ will not be supposed to be inf-compact (nor $C$ to be compact), infimum values (global or local) may not be attained. To be able to deal with this general setting we introduce the notion of local infimum value for the pair $(f, C)$.

DEFInItion 2.1: Let $f: X \rightarrow \mathbb{R} \cup\{+\infty\}$ be a lower semicontinuous function and $C \subset X$ be a nonempty subset of $X$. A real $\lambda$ is called a local infimum value for the pair $(f, C)$ if there exist $\delta>0$ and an open subset $U$ of $X$ such that

$$
\lambda=\inf _{x \in U \cap C} f(x)=\inf _{x \in B_{\delta}(U) \cap C} f(x) .
$$

The set of all local infimum values for $(f, C)$ will be denoted by $\Lambda$. Clearly the image of any local minimiser, if exists, is a local infimum value.

Throughout this paper we shall suppose that $\operatorname{dom} f \cap C \neq \emptyset$ and

$$
m=\inf _{c \in C} f(c)
$$

is finite, that is $f$ is bounded below on $C$. Consequently $\Lambda$ is nonempty and $m$ is the minimal element of $\Lambda$.

One of the most powerfull result concerning the minimisation of a lower semicontinuous function in a complete space is the variational principle of Ekeland [14]. Some "smooth versions" of this principle have been proved in [7] and [4] for unconstrained problems and in [5] for constrained problems using a global concept $r_{C}$ of infimum value. In the same spirit, but based on the concept of local infimum value, we establish a constrained variational principle which will be useful in the sequel.

THEOREM 2.2. Let $f: \mathbb{R}^{n} \rightarrow \mathbb{R} \cup\{+\infty\}$ be a lower semicontinuous function and $C$ be a closed subset of $\mathbb{R}^{n}$. Let $\lambda$ be a local infimum value (associated to $\delta>0$ and an open subset $U$ of $\mathbb{R}^{n}$ ). 
Let us suppose that $U \cap C$ is convex and that $x_{0} \in \mathbb{R}^{n}$ and $\varepsilon>0$ satisfy

$$
x_{0} \in \bar{U} \cap C \text { and } f\left(x_{0}\right)<\lambda+\varepsilon .
$$

Then for any $\eta>0$ such that $f$ is bounded below on $B_{\eta}\left(x_{0}\right)$, there exist $\bar{x} \in B_{\eta}\left(x_{0}\right)$ and $K>0$ such that

$$
\begin{gathered}
f(\bar{x})<f\left(x_{0}\right)+\varepsilon \\
0 \in \partial f(\bar{x})+K \partial d_{U n C}^{2}(\bar{x})+2 \frac{\varepsilon}{\eta} B^{*} .
\end{gathered}
$$

REMARK 2.2.1. Using the same proof, one can obtain this result for any subdifferential satisfying the three properties defining the abstract subdifferential of [5]. In this case, some smoothness of the norm is needed

Proof: Let $\left.\varepsilon^{\prime} \in\right] 0, \varepsilon\left[\right.$ and $\left.\delta^{\prime} \in\right] 0, \delta[$ be such that

$$
f\left(x_{0}\right)<\inf _{U \cap C} f+\varepsilon^{\prime}=\inf _{\bar{B}_{\delta^{\prime}}(U) \cap C} f+\varepsilon^{\prime} .
$$

Let us denote by $A$ the closed subset $A=\bar{U} \cap C$ and by $A_{\eta}$ the subset $A_{\eta}=\bar{B}_{\eta}\left(x_{0}\right) \cap A$. Accordingly to (2) we have $f(x)>f\left(x_{0}\right)-\varepsilon^{\prime}$, for any $x \in A$ and thus, using the lower semicontinuity of $f$ and the compactness of $A_{\eta}$, there exists $\tilde{\delta}>0$ satifying

$$
f\left(x_{0}\right)<f(x)+\varepsilon^{\prime}
$$

for any $x \in \bar{B}_{\tilde{\delta}}\left(A_{\eta}\right)$.

Now we shall denote by $\bar{\delta}$ the real $\bar{\delta}=\inf \left\{\delta^{\prime}, \widetilde{\delta} / 2\right\}$ and by $B$ the closed subset $\bar{B}_{\eta}\left(x_{0}\right) \cup\left[\bar{B}_{\bar{\delta}}(A) \cap C\right]$. Then we define the lower semicontinuous function $g=f+K d_{A}^{2}$ with $K>0$ satisfying

$$
f\left(x_{0}\right)<\inf _{B} f+K \bar{\delta}^{2}+\varepsilon^{\prime}
$$

We claim that $g\left(x_{0}\right)<\inf _{B} g+\varepsilon$. Indeed, if $x \in \bar{B}_{\bar{\delta}}(A) \cap C$ then, according to (2), we have

$$
g\left(x_{0}\right)=f\left(x_{0}\right)<\inf _{\bar{B}_{\mathcal{\delta}}(A) \cap C} f+\varepsilon^{\prime} \leqslant f(x)+\varepsilon^{\prime} \leqslant g(x)+\varepsilon^{\prime} .
$$

Now if $x \in \bar{B}_{\eta}\left(x_{0}\right) \backslash \bar{B}_{\bar{\delta}}(A)$, formula (4) immediately yields

$$
g\left(x_{0}\right)<\inf _{B} f+K \bar{\delta}^{2}+\varepsilon^{\prime} \leqslant f(x)+K d_{A}^{2}(x)+\varepsilon^{\prime}=g(x)+\varepsilon^{\prime} .
$$

Finally, let us suppose that $x$ is an element of $\bar{B}_{\eta}\left(x_{0}\right) \cap \bar{B}_{\bar{\delta}}(A)$. Due to the definition of $\bar{\delta}, x$ is an element of $\bar{B}_{\eta}\left(x_{0}\right) \cap \bar{B}_{\tilde{\delta} / 2}(A)$. We shall show that $x$ is in fact in $\bar{B}_{\tilde{\delta}}\left(A_{\eta}\right)$.

Let $x_{A} \in A$ be such that $\left\|x-x_{A}\right\| \leqslant \tilde{\delta} / 2$. Since $\bar{B}_{\eta}\left(x_{0}\right) \cap \bar{B}_{\tilde{\delta} / 2}(A)$ is nonempty, there exists $\bar{x}_{A} \in\left[x_{0}, x_{A}\right]$ such that $\left\|\bar{x}_{A}-x_{0}\right\| \leqslant \eta$ and $\left\|\bar{x}_{A}-x_{A}\right\| \leqslant \widetilde{\delta} / 2$. Indeed the existence 
of a point $y \in\left[x_{0}, x_{A}\right]$ which is neither in $\bar{B}_{\eta}\left(x_{0}\right)$ nor in $\bar{B}_{\tilde{\delta} / 2}(A)$ leads to the following contradiction

$$
\eta+\tilde{\delta} / 2<\left\|y-x_{0}\right\|+\left\|y-x_{A}\right\|=\left\|x_{0}-x_{A}\right\| \leqslant\left\|x_{0}-x\right\|+\left\|x-x_{A}\right\| \leqslant \eta+\widetilde{\delta} / 2 .
$$

Due to the convexity of $A$ the point $\bar{x}_{A}$ is also an element of $A_{\eta}=\bar{B}_{\eta}\left(x_{0}\right) \cap A$ and

$$
d\left(x, A_{\eta}\right) \leqslant\left\|x-x_{A}\right\|+\left\|x_{A}-\bar{x}_{A}\right\| \leqslant \tilde{\delta} .
$$

Thus, according to inequality (3), $g\left(x_{0}\right)<f(x)+\varepsilon^{\prime} \leqslant g(x)+\varepsilon^{\prime}$ and the claim is proved.

Now applying the Approximate Minimum Theorem ([4, Theorem 3.1]), there exists $\bar{x} \in B_{\eta}\left(x_{0}\right)$ such that

$$
g(\bar{x})<\inf _{B} g+\varepsilon \quad \text { and } \quad 0 \in \partial g(\bar{x})+2(\varepsilon / \eta) B^{*} .
$$

This immediately implies that

$$
f(\bar{x})<g(\bar{x})<\inf _{B} g+\varepsilon \leqslant f\left(x_{0}\right)+\varepsilon .
$$

and using classical subdifferential calculus rules we obtain the announced subdifferential inclusion.

\section{STATIONARY AND MINIMISING SEQUENCES}

Following [2], a sequence $\left(x_{n}\right)_{n}$ is said to be feasible if each $x_{n}$ is an element of $C$ and asymptotically feasible if $\lim _{n \rightarrow \infty} d\left(x_{n}, C\right)=0$.

DEFINITION 3.1: A sequence $\left(x_{n}\right)_{n} \subset \operatorname{dom} f$ is said to be locally minimising if the limit of any converging subsequence of $\left(f\left(x_{n}\right)\right)_{n}$ is an element of $\bar{\Lambda}$.

Clearly when $\Lambda=\{m\}$ (for example whenever $f$ is convex and $C=X$ ) we recover, up to subsequences, the classical definition of minimising sequence (see $[2,3])$.

In the differentiable case, the classical Fermat rule (first order necessary condition) of optimality is

$$
\bar{x} \text { local minimum of } f \text { on } C \Rightarrow\langle\nabla f(\bar{x}), d\rangle \geqslant 0, \quad \forall d \in T_{C}(\bar{x})
$$

where $T_{C}(\bar{x})=\operatorname{cl}\left(\bigcup_{\lambda>0} \lambda(C-\{x\})\right)$ is the Bouligand tangent cone to $C$ at $\bar{x}$. Denoting by $N_{C}(\bar{x})$ the normal cone associated with $T_{C}(\bar{x})$, relation (5) becomes

$$
0 \in \nabla f(\bar{x})+N_{C}(\bar{x}) .
$$

On the other hand, for nonsmooth convex functions and for unconstrained optimisation problems, the necessary condition of optimality $0 \in \partial f(\bar{x})$ leads to the following definition of stationary sequence $([2,3])$ : 


$$
\left(x_{n}\right)_{n} \text { is a stationary sequence if and only if } \lim _{n \rightarrow+\infty} d\left(0, \partial f\left(x_{n}\right)\right)=0 .
$$

To be able to cover nonsmooth and/or nonconvex constrained optimisation problems we propose the following adaptation of the previous points of view:

For any $\varepsilon>0$ let us denote by

$$
T_{C}^{\varepsilon}(x)=\operatorname{cl}\left(\left\{\alpha(c-x): \alpha>0, c \in B_{\varepsilon}(x) \cap C\right\}\right)
$$

the $\varepsilon$-tangent cone to subset $C$ at $x$ and by $N_{C}^{\varepsilon}$ the associated normal cone

$$
N_{C}^{\varepsilon}(x)=\left\{x^{*} \in X^{*}:\left\langle x^{*}, d\right\rangle \leqslant 0, \quad \forall d \in T_{C}^{\varepsilon}(x)\right\} .
$$

For any nonempty convex subset $C$ of $X$, any $x \in C$ and any $\varepsilon>0, T_{C}^{\varepsilon}(x)$ coincides with the Bouligand tangent cone $T_{C}(x)$. But one of the interesting points concerning the definition of $T_{C}^{\varepsilon}(x)$ (and the forthcoming Definition 3.2) is that it allows us to consider non feasible points. Indeed it is not necessary to assume that $x \in \bar{C}$ to have a nonempty tangent cone $T_{C}^{\varepsilon}(x)$.

DEFinition 3.2: A sequence $\left(x_{n}\right)_{n}$ of elements of dom $\partial f$ is said to be a stationary sequence for the couple $(f, C)$ if $\left(x_{n}\right)_{n}$ is asymptotically feasible and there exists $\varepsilon>0$ such that

$$
\lim _{n \rightarrow+\infty} d\left(0, \partial f\left(x_{n}\right)+N_{C}^{\varepsilon}\left(x_{n}\right)\right)=0 .
$$

The choice, in this definition, of a common $\varepsilon$ for any $n$ (instead of a sequence $\varepsilon_{n} \rightarrow 0^{+}$) is guided by the "uniformity" (fixed $\varepsilon$ ) of the concept of local infimum value.

The standard way to reduce the study of constrained optimisation problems to the study of unconstrained ones is to add to the objective function $f$ the indicator function $\Psi_{C}$ of the subset $C$ (that is, $\Psi_{C}(x)=0$ if $x \in C$ and $+\infty$ otherwise). Then an equivalent form of the global problem (1) is

$$
m=\inf _{x \in X}\left(f+\Psi_{C}\right)(x),
$$

and one can define, as in [15], a stationary sequence to be a feasible sequence satisfying

$$
\lim _{n \rightarrow+\infty} d\left(0, \partial\left(f+\Psi_{C}\right)\left(x_{n}\right)\right)=0
$$

or, using some subdifferential calculus rules,

$$
\lim _{n \rightarrow+\infty} d\left(0, \partial f\left(x_{n}\right)+N_{C}^{\partial}\left(x_{n}\right)\right)=0
$$

where $N_{C}^{\partial}$ denote the concept of normal cone associated to the subdifferential $\partial$ (Clarke normal cone $N_{C}^{c r}$ for Clarke-Rockafellar subdifferential, Bouligand normal cone $N_{C}$ for Hadamard subdifferential and so on). To be able to deal with possibly non feasible sequences one can replace $\Psi_{C}$ by the distance function $d_{C}$ and (8) becomes

$$
\lim _{n \rightarrow+\infty} d\left(0, \partial f\left(x_{n}\right)+\partial d_{C}\left(x_{n}\right)\right)=0
$$


But none of those approaches is sharp enough to study the relation between stationary and minimising sequences. For example in the very simple case of the minimisation of the real function $f(x, y)=y-x$ on the subset $C=\{0\} \times[0,1]$ of $\mathbb{R}^{2}$, the sequence $\left(x_{n}\right)_{n}=((0,-1 / n))_{n}$ is minimising and stationary (in the sense of Definition 3.2) while the only sequence satisfying (8) is the null sequence. Observe that the sequence $\left(x_{n}\right)_{n}$ does not either satisfy relation (9).

Nevertheless every feasible stationary sequence in the sense of Defintion 3.2 satisfies (8). Indeed, from the definition of $T_{C}^{\varepsilon}$ it is clear that for any subset $C \subset X$ and any $x \in C$ one has $T_{C}(x) \subset T_{C}^{\varepsilon}(x)$ and thus $d\left(0,\left(\partial f+N_{C}\right)(x)\right) \leqslant d\left(0,\left(\partial f+N_{C}^{\varepsilon}\right)(x)\right)$. The reverse implication does not hold, in general. For example consider the linear function $f: \mathbb{R}^{2} \rightarrow \mathbb{R}$ defined by $f(x, y)=x$ and the compact subset

$$
C=\{(x,-\sqrt{x}): 0 \leqslant x \leqslant 1\} \cup([-2,0] \times[-1,0]) .
$$

Then one can prove that every feasible stationary sequence is a minimising sequence but there exists feasible sequences satisfying (8) (and (9)) (for example $(1 / n,-\sqrt{1 / n})_{n}$ ) which are not minimising sequences.

Actually, Definition 3.2 can be seen as a first step in what could be called a "mixed nonsmooth optimality condition" in which the concept of normal cone considered for the epigraph of the objective function (and thus the concept of subdifferential) and the concept of normal cone considered for the constraint set are chosen independently.

In the following result we show that, roughly speaking, in the neighbourhood of every feasible minimising sequence converging to a local infimum value there exists a minimising sequence which is also a stationary sequence.

TheOREM 3.3. Let $f: \mathbb{R}^{n} \rightarrow \mathbb{R}$ be a Lipschitz function and $C$ be a closed nonempty subset of $\mathbb{R}^{n}$. Let $\left(x_{n}\right)_{n}$ be a feasible sequence such that $\lambda=\lim _{n \rightarrow \infty} f\left(x_{n}\right)$ is a local infimum value (associated to the open subset $U$ and the positive real $\delta$ ).

Suppose that $U \cap C$ and $B_{\delta}(U) \cap C$ are convex, $f$ is bounded below on $B_{\delta}(U)$ and $\lim _{n \rightarrow+\infty} d\left(x_{n}, U \cap C\right)=0$.

Then there exist a subsequence $\left(x_{n_{k}}\right)_{k}$ of $\left(x_{n}\right)_{n}$ and a stationary and minimising sequence $\left(y_{k}\right)_{k}$ of elements of $\mathbb{R}^{n}$ such that

$$
\lim _{k \rightarrow+\infty}\left\|y_{k}-x_{n_{k}}\right\|=0 \quad \text { and } \quad \lim _{k \rightarrow+\infty} f\left(y_{k}\right)=\lambda .
$$

Lemma 3.4. Let $C$ be a nonempty subset of $\mathbb{R}$ and $f: \mathbb{R}^{n} \rightarrow \mathbb{R}$. Let $\lambda$ be a local infimum value for $(f, C)$, associated to the open subset $U$ and to the real $\delta>0$. Then, for any $\left.\delta^{\prime} \in\right] 0, \delta\left[\right.$ there exists $\delta^{\prime \prime}>0$ such that

$$
\lambda=\inf _{A \cap C} f=\inf _{B_{\delta^{\prime \prime}}(A) \cap C} f
$$

where $A=\bar{B}_{\delta^{\prime}}(\bar{U} \cap C)$. 
PROOF: Let $\delta^{\prime}$ be an element of $] 0, \delta\left[\right.$ and $\left.\delta^{\prime \prime} \in\right] 0, \delta-\delta^{\prime}[$. We immediately obtain that any $x \in B_{\delta^{\prime \prime}}(A) \cap C$ is an element of $B_{\delta}(U) \cap C$ since $d(x, U) \leqslant d(x, U \cap C)<\delta^{\prime}+\delta^{\prime \prime}<\delta$. On the other hand $(U \cap C) \subset(A \cap C)$. The desired equalities are a direct consequence of those inclusions.

Proof of Theorem 3.3: Let $\left.\delta^{\prime} \in\right] 0, \delta\left[\right.$. From Lemma 3.4 there exists $\delta^{\prime \prime}>0$ such that

$$
\lambda=\inf _{A \cap C} f=\inf _{B_{\delta^{\prime \prime}}(A) \cap C} f,
$$

where $A=\bar{B}_{\delta^{\prime}}(\bar{U} \cap C)$. Let us observe that $A \cap C=B_{\delta^{\prime}}(U \cap C) \cap\left[B_{\delta}(U) \cap C\right]$ is a convex subset of $\mathbb{R}^{n}$, as it is the intersection of two convex subsets.

Let $\left(x_{n_{k}}\right)_{k}$ be a subsequence of $\left(x_{n}\right)_{n}$ such that, for any $k \in \mathbb{N}$

$$
f\left(x_{n_{k}}\right)<\lambda+1 / k^{2} .
$$

For $k$ large enough (say $\left.k \geqslant k_{0}\right) x_{n_{k}} \in\left[B_{\delta^{\prime} / 3}(U \cap C) \cap C\right] \subset(A \cap C)$ and thus, applying Theorem 2.2 with $x_{0}=x_{n_{k}}, U=A, \varepsilon=1 / k^{2}$ and $\eta=1 / k$ there exist $y_{k} \in B_{1 / k}\left(x_{n_{k}}\right)$ and $R_{k}>0$ satisfying

$$
\begin{gathered}
f\left(y_{k}\right)<f\left(x_{n_{k}}\right)+1 / k^{2}, \\
0 \in \partial f\left(y_{k}\right)+R_{k} \partial d_{A \cap C}^{2}\left(y_{k}\right)+2 / k B^{*} .
\end{gathered}
$$

According to (11) one can find $y_{k}^{*} \in \partial f\left(y_{k}\right), \alpha_{k}^{*} \in \partial d_{A \cap C}^{2}\left(y_{k}\right)$ and $\beta_{k}^{*} \in B^{*}$ satisfying $y_{k}^{*}+R_{k} \alpha_{k}^{*}=2 / k \beta_{k}^{*}$.

On the other hand, for $k$ large enough $\left(k \geqslant k_{1} \geqslant k_{0}\right), B_{\delta^{\prime} / 3}\left(y_{k}\right) \subset A$ and thus,

$$
\left\langle\alpha_{k}^{*}, c-y_{k}\right\rangle \leqslant 0, \quad \forall c \in B_{\delta^{\prime} / 3}\left(y_{k}\right) \cap C
$$

since $A \cap C$ is convex. Consequently, for any $k \geqslant k_{1}, y_{k}^{*}+R_{k} \alpha_{k}^{*}$ is an element of $\partial f\left(y_{k}\right)$ $+N_{C}^{\delta^{\prime} / 3}\left(y_{k}\right)$ and $\lim _{k \rightarrow \infty} y_{k}^{*}+R_{k} \alpha_{k}^{*}=0$.

The sequence $\left(y_{k}\right)_{k}$ is therefore a stationary sequence. But it is also a minimising sequence since, according to (10) and to the Lipschitz continuity of $f$ (with Lipschitz $\operatorname{rank} K_{f}$ )

$$
f\left(x_{n_{k}}\right)-K_{f} / k \leqslant f\left(y_{k}\right)<f\left(x_{n_{k}}\right)+1 / k^{2}<\lambda+2 / k^{2} .
$$

\section{Constrained asymptotical Well Behaviour}

Based on the previous definitions, the two main results of this last section (Theorems 4.4 and 4.7) establish respectively a sufficient and a necessary condition for a couple $(f, C)$ to have "constrained asymptotical well behaviour", that is, any stationary sequence is a locally minimising sequence. 
Definition 4.1: Let $f: X \rightarrow \mathbb{R} \cup\{+\infty\}$ be a lower semicontinuous function and $C$ be a nonempty subset of $X$. The couple $(f, C)$ will be said to have a constrained asymptotical well behaviour if every stationary sequence is a locally minimising sequence.

Let us denote by $\mathcal{F}_{c}$ (respectively by $\mathcal{F}_{c}^{s}$ ) the set of couples $(f, C$ ), with $f$ lower semicontinuous and $C$ closed nonempty subset of $X$, such that

For any compact segment $J$ of $\mathbb{R}$ with $J \cap \bar{\Lambda}=\emptyset$ one has

$$
\inf _{\substack{\lambda \in J \in C \backslash L(\lambda) \\ f(x) \in J}} \frac{f(x)-\lambda}{d(x, L(\lambda) \cap C)}>0
$$

respectively

$$
\left.\inf _{\lambda \in J \in J \backslash L(\lambda)} \inf _{\substack{x \in(x) \in J \\ f(x)}} \frac{f(x)-\lambda}{d(x, L(\lambda) \cap C)}>0\right)
$$
$(f, C)$.

Clearly $\mathcal{F}_{c}^{s}$ is a subset of $\mathcal{F}_{c}$ and corresponds to a stronger assumption on the couple

Those definitions are some natural extensions of the following property used in [3] to characterise, for unconstrained optimisation problems, the set $\mathcal{F}$ of convex functions for which every stationary sequence is a minimising sequence:

$$
f \in \mathcal{F} \Leftrightarrow \forall \lambda>m, \quad l(\lambda)=\inf _{x \in X \backslash L(\lambda)} \frac{f(x)-\lambda}{d(x, L(\lambda))}>0 .
$$

Proposition 4.2. Let $f: X \rightarrow \mathbb{R} \cup\{+\infty\}$ be a proper lower semicontinuous convex function. Then

$$
f \in \mathcal{F} \Longleftrightarrow(f, X) \in \mathcal{F}_{c} \Longleftrightarrow(f, X) \in \mathcal{F}_{c}^{s}
$$

Proof: The implication $f \in \mathcal{F} \Longrightarrow(f, X) \in \mathcal{F}_{c}$ is a direct consequence of the increasing property of $l$ (see [3, Proposition 6.2 and 6.3]), that is, $l\left(\lambda^{\prime}\right) \geqslant l(\lambda)$ for any $\lambda^{\prime} \geqslant \lambda$.

For the reverse implication, suppose that $(f, X) \in \mathcal{F}_{c}$ and $\lambda>\inf _{X} f$ and consider $J=[\lambda, \lambda+1]$. Then $f$ is an element of $\mathcal{F}$ since

$$
\inf _{\substack{x \notin L(\lambda) \\ f(x) \in J}} \frac{f(x)-\lambda}{d(x, L(\lambda))}>\inf _{\nu \in J} \inf _{\substack{x \notin L(\nu) \\ f(x) \in J}} \frac{f(x)-\nu}{d(u, L(\nu))}>0
$$

and, for any $x \notin L(\lambda)$ with $f(x) \notin J$ one clearly have, using the convexity of $f$,

$$
\frac{f(x)-\lambda}{d(x, L(\lambda))} \geqslant \inf _{\substack{u \notin L(\lambda) \\ f(u) \in J}} \frac{f(u)-\lambda}{d(u, L(\lambda))}
$$


Let us recall that a function $f: X \rightarrow \mathbb{R} \cup\{+\infty\}$ is said to be weakly convex provided that there exists $\rho \geqslant 0$ such that, for any $x, y \in \operatorname{dom} f$ and any $t \in[0,1]$ one has

$$
f(t x+(1-t) y) \leqslant t f(x)+(1-t) f(y)+\rho t(1-t)\|y-x\|^{2} .
$$

The weak convexity has been introduced by Janin [16] (in a local form) postulating that the function $f$ can be decomposed into the sum of a convex function and $\rho\|\cdot\|^{2}$. If $X$ is an Hilbert space this definition is equivalent to the previous one (see $[24,8]$ ). Weakly convex functions are particular cases of lower- $C^{2}$ functions and have been extensively studied (Cornet [10], Cornet and Vial [11], Rockafellar [23], Vial [24], for example). Let us observe that any weakly convex function is Lipschitz continuous on its domain (see [24]).

As a straightforward extension of [24, Proposition 4.8] we obtain

Proposition 4.3. Let $X$ be an Hilbert space and $f: X \rightarrow \mathbb{R} \cup\{+\infty\}$ be a function with convex domain.

Then $f$ is $\rho$-weakly convex if and only if for any $x, y \in \operatorname{dom} f$ and any $x^{*} \in \partial f(x)$, one has

$$
\left\langle x^{*}, y-x\right\rangle \leqslant f(y)-f(x)+\rho\|y-x\|^{2} .
$$

We are now in position to state our main result concerning constrained asymptotical well behaviour of a nonconvex optimisation problem.

THEOREM 4.4. Let $C$ be a closed nonempty subset of an Hilbert space $X$ and $f: X \rightarrow \mathbb{R}$ be a lower semicontinuous weakly convex function.

(i) If $(f, C) \in \mathcal{F}_{c}^{s}$ then any stationary sequence is a locally minimising sequence

(ii) If $(f, C) \in \mathcal{F}_{c}$ then any feasible stationary sequence is a locally minimising sequence.

Moreover, if $f$ is convex, assertions (i) and (ii) hold true for any real Banach space $X$.

Let us first prove the following lemma.

LEMMA 4.5. Let $C$ be a closed nonempty subset of an Banach space $X$ and $f: X \rightarrow \mathbb{R}$ be a Lipschitz function. Let $\left(x_{n}\right)_{n}$ be an asymptotically feasible sequence such that $\lambda=\lim _{n \rightarrow \infty} f\left(x_{n}\right) \notin \bar{\Lambda}$ with $\lambda \in \mathbb{R}$.

If $(f, C) \in \mathcal{F}_{c}^{s}$ or if $(f, C) \in \mathcal{F}_{c}$ and $\left(x_{n}\right)_{n}$ is feasible, then there exist $\mu>0$, a subsequence $\left(x_{n_{k}}\right)_{k}$ of $\left(x_{n}\right)_{n}$ and a sequence $\left(y_{k}\right)_{k}$ of elements of $C$ satisfying $\lim _{k \rightarrow \infty} \| x_{k}$ $-y_{k} \|=0$ and, for any $k$,

$$
0<\mu\left\|x_{n_{k}}-y_{k}\right\| \leqslant f\left(x_{n_{k}}\right)-f\left(y_{k}\right)
$$

Proof: (i) Let us suppose first that $(f, C) \in \mathcal{F}_{c}^{s}$. It is sufficient to consider the following situations: $\left(f\left(x_{n}\right)\right)_{n}$ decreasing or $\left(f\left(x_{n}\right)\right)_{n}$ strictly increasing. 
Let $\eta>0$ be such that $J \cap \Lambda=\emptyset$ where $J=[\lambda-\eta, \lambda+\eta]$.

CASE A. $\left(f\left(x_{n}\right)\right)_{n}$ is decreasing.

Fix $\varepsilon>0$ and define, for any $n$, the (possibly infinite) value $\varepsilon_{n}=d\left(x_{n}, M_{n}\right)$ with

$$
M_{n}=\left\{x \in B_{\varepsilon}\left(x_{n}\right) \cap C: f(x)<f\left(x_{n}\right)\right\} .
$$

Claim. the sequence $\left(\varepsilon_{n}\right)_{n}$ admits a subsequence $\left(\varepsilon_{n_{k}}\right)_{k}$ converging to 0 .

Indeed, let us suppose, for a contradiction, that there exists $\beta>0$ such that $\varepsilon_{n}$ $=d\left(x_{n}, M_{n}\right) \geqslant \beta$, for any $n$. Note that in this case some subset $M_{n}$ could be empty.

If we denote by $\alpha$ the real $\alpha=\min \{\varepsilon, \beta\}$ then for any $n$ and any $x \in B_{\alpha}\left(x_{n}\right) \cap C$ one has $f(x) \geqslant f\left(x_{n}\right)$. Since $\left(x_{n}\right)_{n}$ is an asymptotically feasible sequence, for $n$ large enough (say $\left.n \geqslant n_{0}\right) B_{\alpha / 2}\left(x_{n}\right) \cap C$ is a nonempty subset. So one can find, for $n \geqslant n_{0}$, $u_{n} \in B_{\alpha / 2}\left(x_{n}\right) \cap C$ and $v_{n} \in B_{\alpha}\left(x_{n}\right) \cap C$ verifying

$$
f\left(u_{n}\right) \geqslant \inf _{x \in B_{\alpha / 2}\left(x_{n}\right) \cap C} f(x) \geqslant f\left(x_{n}\right), \quad f\left(v_{n}\right) \geqslant \inf _{x \in B_{\alpha}\left(x_{n}\right) \cap C} f(x) \geqslant f\left(x_{n}\right)
$$

with

$$
\left\|x_{n}-u_{n}\right\| \leqslant d\left(x_{n}, C\right)+1 / n \text { and }\left\|x_{n}-v_{n}\right\| \leqslant d\left(x_{n}, C\right)+1 / n
$$

Denoting by $U$ the open subset $U=\bigcup_{n \geqslant n_{0}} B_{\alpha / 2}\left(x_{n}\right)$, we obtain, for $n \geqslant n_{0}$,

$$
f\left(u_{n}\right) \geqslant \inf _{x \in U \cap C} f(x) \geqslant \inf _{k \geqslant n_{0}} f\left(x_{k}\right)=\lambda
$$

and

$$
f\left(v_{n}\right) \geqslant \inf _{x \in B_{\alpha / 2}(U) \cap C} f(x) \geqslant \inf _{k \geqslant n_{0}} f\left(x_{k}\right)=\lambda .
$$

But $f$ is a Lipschitz continuous function and thus, using (15), the sequences $\left(f\left(u_{n}\right)\right)_{n}$ and $\left(f\left(v_{n}\right)\right)_{n}$ converge to $\lambda$. This leads us to the desired contradiction since, together with (16) and (17), we can conclude that $\lambda=\inf _{x \in U \cap C} f(x)=\inf _{x \in B_{\alpha / 2}(U) \cap C} f(x)$ is an element of $\Lambda$. Thus the claim is proved.

According to our claim let $\left(\varepsilon_{n_{k}}\right)_{k}$ be a subsequence of $\left(\varepsilon_{n}\right)_{n}$ converging to 0 and, for any $k$, let $\widehat{x}_{k} \in B_{\varepsilon}\left(x_{n_{k}}\right) \cap C$ be such that

$$
f\left(\widehat{x}_{k}\right)<f\left(x_{n_{k}}\right) \text { and }\left\|\widehat{x}_{k}-x_{n_{k}}\right\| \leqslant \varepsilon_{n_{k}}+1 / n_{k} .
$$

Since $f$ is Lipschitz continuous, one has

$$
\lim _{k \rightarrow \infty} f\left(\widehat{x}_{k}\right)=\lambda
$$


and thus, for $k$ large enough (say $\left.k \geqslant k_{0}\right) f\left(\widehat{x}_{k}\right)$ is an element of $J$. On the other hand $x_{n_{k}} \notin L\left(f\left(\widehat{x}_{k}\right)\right)$. From the definition of $\mathcal{F}_{c}^{s}$ (used with $J$ ) there exists $\mu>0$ such that, for $k \geqslant k_{0}$,

$$
f\left(x_{n_{k}}\right)-f\left(\widehat{x}_{k}\right) \geqslant \mu d\left(x_{n_{k}}, L\left(f\left(\widehat{x}_{k}\right)\right) \cap C\right) .
$$

Now considering $\left.\mu^{\prime} \in\right] 0, \mu\left[\right.$, one can construct a sequence $\left(y_{k}\right)_{k}$ of elements of $C$ satisfying, for $k \geqslant k_{0}, y_{k} \in L\left(f\left(\widehat{x}_{k}\right)\right) \cap C$ and

$$
f\left(x_{n_{k}}\right)-f\left(y_{k}\right) \geqslant \mu d\left(x_{n_{k}}, L\left(f\left(\widehat{x}_{k}\right)\right) \cap C\right) \geqslant \mu^{\prime}\left\|y_{k}-x_{n_{k}}\right\|>0, \quad \forall k \geqslant k_{0} .
$$

Finally, the previous inequality, together with (18) and (19) immediately implies

$$
\lim _{k \rightarrow \infty}\left\|x_{n_{k}}-y_{k}\right\|=0
$$

CAse B. $\left(f\left(x_{n}\right)\right)_{n}$ is strictly increasing. From the definition of $\mathcal{F}_{c}^{s}$ with $J=[\lambda-\eta, \lambda+\eta]$ one can find a constant $\mu>0$ such that for every $\nu \in J$ and every $x \notin L(\nu)$, one has

$$
\frac{f(x)-\nu}{d(x, L(\nu) \cap C)} \geqslant \mu \text {. }
$$

Since, for $n$ large enough, $f\left(x_{n}\right) \in J$ and $x_{n+1} \notin L\left(f\left(x_{n}\right)\right)$ we obtain

$$
\mu d\left(x_{n+1}, L\left(f\left(x_{n}\right)\right) \cap C\right) \leqslant f\left(x_{n+1}\right)-f\left(x_{n}\right) .
$$

Let $\mu^{\prime} \in \mathbb{R}$ be such that $0<\mu^{\prime}<\mu$ and denote by $y_{n+1}$ an element of $L\left(f\left(x_{n}\right)\right) \cap C$ such that

$$
\mu^{\prime}\left\|x_{n+1}-y_{n+1}\right\|<\mu d\left(x_{n+1}, L\left(f\left(x_{n}\right)\right) \cap C\right) .
$$

Then, as in the previous case, by (20) and (21) we obtain, for any $n \geqslant n_{0}$

$$
\mu^{\prime}\left\|x_{n+1}-y_{n+1}\right\| \leqslant f\left(x_{n+1}\right)-f\left(x_{n}\right) \leqslant f\left(x_{n+1}\right)-f\left(y_{n+1}\right)
$$

and therefore $\lim _{n \rightarrow+\infty}\left\|x_{n+1}-y_{n+1}\right\|=0$.

(ii) The same proof remains valid if $(f, C)$ is only supposed to be an element of $\mathcal{F}_{c}$, providing that the sequence $\left(x_{n}\right)_{n}$ is feasible (and invoking definition (12) instead of (13)).

Proof of THEOREM 4.4: Let $\left(x_{n}\right)_{n}$ be a stationary sequence for $(f, C)$ such that $\left(f\left(x_{n}\right)\right)_{n}$ converge (to $\left.\lambda \in \mathbb{R}\right)$. Thus there exist $\varepsilon>0$ and, for any $n, x_{n}^{*} \in \partial f\left(x_{n}\right)$ and $w_{n}^{*} \in N_{C}^{\varepsilon}\left(x_{n}\right)$ such that

$$
\lim _{n \rightarrow \infty}\left(x_{n}^{*}+w_{n}^{*}\right)=0
$$


Now if $\lambda \notin \bar{\Lambda}$ then, according to Lemma 4.5 and Proposition 4.3 (or the definition of the Fenchel subdifferential if $f$ is convex), there exist $\mu>0$, a subsequence $\left(x_{n_{k}}\right)_{k}$ of $\left(x_{n}\right)_{n}$ and a sequence $\left(y_{k}\right)_{k}, y_{k} \in C$ such that

$$
\lim _{k \rightarrow \infty}\left\|x_{n_{k}}-y_{k}\right\|=0
$$

and, for any $k$,

$$
\left\langle x_{n_{k}}^{*}, y_{k}-x_{n_{k}}\right\rangle \leqslant-\mu\left\|y_{k}-x_{n_{k}}\right\|+\rho\left\|y_{k}-x_{n_{k}}\right\|^{2} .
$$

Finally, for $k$ large enough, $y_{k} \in B_{\varepsilon}\left(x_{n_{k}}\right) \cap C$ and

$$
\mu \leqslant \frac{\left\langle-\left(x_{n_{k}}^{*}+w_{n_{k}}^{*}\right), y_{k}-x_{n_{k}}\right\rangle}{\left\|y_{k}-x_{n_{k}}\right\|}+\rho\left\|y_{k}-x_{n_{k}}\right\| \leqslant\left\|x_{n_{k}}^{*}+w_{n_{k}}^{*}\right\|+\rho\left\|y_{k}-x_{n_{k}}\right\|
$$

which contradicts (22) or (23).

Item (ii) of Theorem 4.4 only deals with feasible stationary sequences. This could be considered to be quite restrictive. However, as it will be shown by the next proposition, even in a restrictive form (feasible stationary sequence and convex function) Theorem 4.4-(ii) is powerfull enough to obtain asymptotical well behaviour results for classes of nonconstrained optimisation problems which were not covered by previous approaches, since the function is supposed to be neither convex (as in [3]), quasiconvex (as in [19]), nor critical (as in [15]).

For any function $g: \mathbb{R}^{2} \rightarrow \mathbb{R}$ we associate the nonempty subset $C_{g}$ of $\mathbb{R}^{3}$ defined by

$$
C_{g}=\left\{(x, y, z) \in \mathbb{R}^{3}: z=g(x, y)\right\} .
$$

Proposition 4.6. Let $F: \mathbb{R}^{2} \rightarrow \mathbb{R}$ be a differentiable function defined by $F(x, y)=h(x)+g(x, y)$ where $h$ is any differentiable convex function and $g$ is a Lipschitz differentiable function such that there exists $\varepsilon>0$ for which

$$
\left(\frac{\partial g}{\partial x}(x, y), \frac{\partial g}{\partial y}(x, y),-1\right) \in N_{C_{g}}^{\varepsilon}(x, y, g(x, y)), \quad \forall(x, y) \in \mathbb{R}^{2} .
$$

If $\left(F, \mathbb{R}^{2}\right) \in \mathcal{F}_{c}$ then any stationary sequence is a locally minimising sequence for $\left(F, \mathbb{R}^{2}\right)$, that is,

$$
\lim _{n \rightarrow \infty} \nabla F\left(x_{n}, y_{n}\right)=0 \Rightarrow \text { "if } \lambda=\lim _{k \rightarrow+\infty} f\left(x_{n_{k}}\right) \text { then } \lambda \in \bar{\Lambda} " .
$$

Note that since $F$ is not assumed to be convex it becomes important to use the local infimum concept (and thus the subset $\Lambda$ ).

Proof: At first let us define the differentiable convex function $f: \mathbb{R}^{3} \rightarrow \mathbb{R}$ by $f(x, y, z)=h(x)+z$. Let us denote by $\Lambda\left(f, C_{g}\right)$ (respectively $\Lambda(F)$ ) the set of local infimum values associated to the pair $\left(f, C_{g}\right)$ (respectively, the pair $\left.\left(F, \mathbb{R}^{2}\right)\right)$. Clearly, for any $(x, y) \in \mathbb{R}^{2}$ one has $F(x, y)=f(x, y, g(x, y))$ and thus $\Lambda\left(f, C_{g}\right)=\Lambda(F)$. 
On the other hand, since $g$ is Lipschitz of rank $K$ on $\mathbb{R}^{2}$ and, for any $(x, y, z) \in \mathbb{R}^{3}$

$$
f(x, y, z) \leqslant \lambda \text { and }(x, y, z) \in C_{g} \quad \Leftrightarrow \quad F(x, y) \leqslant \lambda
$$

then

$$
d_{\mathbb{R}^{2}}\left((x, y), L_{F}(\lambda)\right) \leqslant d_{\mathbb{R}^{3}}\left((x, y, z), L_{f}(\lambda) \cap C_{g}\right) \leqslant(K+1) d_{\mathbb{R}^{2}}\left((x, y), L_{F}(\lambda)\right)
$$

providing that $(x, y, z)$ is an element of $C_{g}$. As an immediate consequence one obtain, for any $\lambda \in \mathbb{R}$, the equivalence between the inequalities

$$
\inf _{(x, y) \in \mathbb{R}^{2} \backslash L_{F}(\lambda)} \frac{F(x, y)-\lambda}{d\left((x, y), L_{F}(\lambda)\right)}>0
$$

and

$$
\inf _{(x, y, z) \in C_{g} \backslash L_{f}(\lambda)} \frac{f(x, y, z)-\lambda}{d\left((x, y, z), L_{f}(\lambda) \cap C_{g}\right)}>0 .
$$

Finally let us suppose that $\left(\left(x_{n}, y_{n}\right)\right)_{n}$ is a stationary sequence for $\left(F, \mathbb{R}^{2}\right)$, that is,

$$
\lim _{n \rightarrow \infty}\left\|\nabla F\left(x_{n}, y_{n}\right)\right\|=0 \text {. }
$$

Due to the assumption (24) on $g$, there exists $\varepsilon>0$ such that for any $n, V_{n}$ $=\left(\frac{\partial g}{\partial x}\left(x_{n}, y_{n}\right), \frac{\partial g}{\partial y}\left(x_{n}, y_{n}\right),-1\right)$ is an element of $N_{C_{g}}^{\varepsilon}\left(x_{n}, y_{n}, g\left(x_{n}, y_{n}\right)\right)$ and

$$
\lim _{n \rightarrow \infty}\left\|\nabla f\left(x_{n}, y_{n}, g\left(x_{n}, y_{n}\right)\right)+V_{n}\right\|=\lim _{n \rightarrow \infty}\left\|\nabla F\left(x_{n}, y_{n}\right)\right\|=0
$$

By Theorem $4.4-i i)$, the feasible stationary sequence $\left(\left(x_{n}, y_{n}, g\left(x_{n}, y_{n}\right)\right)\right)_{n}$ is a locally minimising sequence for $\left(f, C_{g}\right)$ and thus, using the equivalence between (25) and (26), $\left(\left(x_{n}, y_{n}\right)\right)_{n}$ is a locally minimising sequence for $\left(F, \mathbb{R}^{2}\right)$.

The function $f$ and the subset $C$ will be said to be compatible if there exists $K \geqslant 1$ such that for any $x \in C$ and for any $\lambda \in f(C)$, one has

$$
d(x, L(\lambda) \cap C) \leqslant K d(x, L(\lambda)) .
$$

Such a geometric link between the function and the constraint set is essential in the following result in which we establish a necessary condition for the couple $(f, C)$ to have a constrained asymptotical well behaviour.

TheOREM 4.7. Let $X$ be an Hilbert space, $f: X \rightarrow \mathbb{R}$ be a lower semicontinuous convex function and $C$ be a nonempty closed subset of $X$. Suppose that $f$ and $C$ are compatible and

(a) $\forall \lambda: \inf _{X} f<\lambda<\mu, \quad \exists \varepsilon>0: L(\mu) \subset B_{\varepsilon}(L(\lambda))$ 
(b) $\exists \bar{\varepsilon}>0: \forall x \in C, N_{C}^{c r}(x)=N_{C}^{\bar{\varepsilon}}(x)$.

Then, if $(f, C)$ has a constrained asymptotical well behaviour then $(f, C) \in \mathcal{F}_{c}$.

REMARK 4.7.1. (i) Hypothesis (a) does not implies that the sublevel sets of $f$ are bounded. It is fullfilled, for example, by linear functions and inf-compact functions.

(ii) Assumption (b) is obviously satisfied if $C$ is convex or if $C$ is a countable union of disjoint convex subsets $\left(C_{i}\right)_{i \in \mathbb{N}}$ with $\bar{\varepsilon}=\inf _{i \neq j \in \mathbb{N}} d\left(C_{i}, C_{j}\right)>0$.

Proof: Let us suppose that $(f, C) \notin \mathcal{F}_{c}$. Thus there exist a compact subset $J$ $=[\alpha, \beta]$ of $f(C)$, a sequence $\left(x_{n}\right)_{n} \subset C$ and a sequence $\left(\lambda_{n}\right)_{n} \subset J$ such that

$$
\begin{gathered}
J \cap \bar{\Lambda}=\emptyset \\
f\left(x_{n}\right) \in J \backslash L\left(\lambda_{n}\right) \\
\lim _{n \rightarrow \infty} \frac{f\left(x_{n}\right)-\lambda_{n}}{d\left(x_{n}, L\left(\lambda_{n}\right) \cap C\right)}=0 .
\end{gathered}
$$

One can assume, without loss of generality, that $\lambda_{n}$ converge to an element $\bar{\lambda}$ of $J$. Let us denote by $\Delta_{n}=d\left(x_{n}, L\left(\lambda_{n}\right) \cap C\right)$ and by $\varepsilon_{n}=\left(f\left(x_{n}\right)-\lambda_{n}\right) / \Delta_{n}$.

According to (28) and the compactness of $J$, the sequence $\left(\Delta_{n}\right)_{n}$ is bounded (let say by $M>0)$. Indeed, $\left(x_{n}\right)_{n} \subset L(\beta)$ and, for any $n, L(\alpha) \subset L\left(\lambda_{n}\right) \subset L(\beta)$. But, due to assumption $(a), L(\beta) \subset B_{\varepsilon}(L(\alpha))$ for some $\varepsilon>0$. Thus the sequence $\left(d\left(x_{n}, L\left(\lambda_{n}\right)\right)\right)_{n}$ is bounded as well as $\left(d\left(x_{n}, L\left(\lambda_{n}\right) \cap C\right)\right)_{\mathfrak{n}}$ since $f$ and $C$ are compatible.

The task is now to construct a stationary sequence. Depending on the behaviour of $\left(\Delta_{n}\right)_{n}$ we distinguish two cases:

CASE 1. There exists $\Delta>0$ such that $\Delta_{n}>\Delta, \forall n$.

Then for any $n$,

$$
\begin{aligned}
\left(f+\psi_{C_{n}}\right)\left(x_{n}\right)=f\left(x_{n}\right) & =\lambda_{n}+\varepsilon_{n} \Delta_{n} \\
& \leqslant \lambda_{n}+M \varepsilon_{n} \\
& \leqslant \inf _{x \in X}\left(f+\psi_{C_{n}}\right)(x)+M \varepsilon_{n}
\end{aligned}
$$

where $C_{n}$ denotes the closed subset $C_{n}=\bar{B}_{\Delta}\left(x_{n}\right) \cap C$. Now applying [21, Theorem 4.10] (or rather the extension of this result to the case of Lipschitz and continuously differentiable bump functions, see also [13]) there exist $\bar{x}_{n} \in \bar{B}_{\Delta}\left(x_{n}\right) \cap C$ and a Lipschitz continuously differentiable function $g_{n}: X \rightarrow \mathbb{R}^{+}$satisfying, for $n$ large enough,

$$
\begin{gathered}
d\left(\bar{x}_{n}, x_{n}\right)<\Delta / 2 \\
\left(f+g_{n}\right)\left(\bar{x}_{n}\right)=\inf _{x \in \bar{B}_{\Delta}\left(x_{n}\right) \cap C}\left(f+g_{n}\right)(x) \\
\left\|g_{n}\right\|_{\infty}<\sqrt{M \varepsilon_{n} / a} \text { and }\left\|D g_{n}\right\|_{\infty}<\sqrt{M \varepsilon_{n} / a} ;
\end{gathered}
$$


where $a$ is a positive constant (not depending on $n$ ) defined in [21, Theorem 4.10]. We conclude from (31) and (32) that

$$
\left(f+g_{n}\right)\left(\bar{x}_{n}\right)=\inf _{x \in B_{\bar{\varepsilon}}\left(\bar{x}_{n}\right) \cap C}\left(f+g_{n}\right)(x)
$$

where $\widetilde{\varepsilon}=\min [\Delta / 2, \bar{\varepsilon}]$, and hence, according to assumption $(b)$, that

$$
0 \in \partial\left(f+g_{n}\right)\left(\bar{x}_{n}\right)+N_{B_{\tilde{\varepsilon}}}^{c r}\left(\bar{x}_{n}\right) \cap C\left(\bar{x}_{n}\right) \subset \partial f\left(\bar{x}_{n}\right)+D g_{n}\left(\bar{x}_{n}\right)+N_{C}^{\tilde{\varepsilon}}\left(\bar{x}_{n}\right) \text {. }
$$

Finally $d\left(0, \partial f\left(\bar{x}_{n}\right)+N_{C}^{\tilde{\varepsilon}}\left(\bar{x}_{n}\right)\right) \leqslant\left\|D g_{n}\left(\bar{x}_{n}\right)\right\|_{\infty}$ and thus, using (33) and (29), $\left(\bar{x}_{n}\right)_{n}$ is a feasible stationary sequence and consequently a locally minimising sequence.

On the other hand, due to (30), (31), (32) and (33) one has

$$
\lambda_{n}<f\left(\bar{x}_{n}\right) \leqslant\left(f+g_{n}\right)\left(\bar{x}_{n}\right) \leqslant\left(f+g_{n}\right)\left(x_{n}\right)<\lambda_{n}+M \varepsilon_{n}+\sqrt{M \varepsilon_{n} / a} .
$$

Therefore $\bar{\lambda}=\lim _{n \rightarrow \infty} \lambda_{n}=\lim _{n \rightarrow \infty} f\left(\bar{x}_{n}\right) \in \bar{\Lambda}$ and the desired contradiction is obtained since $J \cap \bar{\Lambda}=\emptyset$.

CASE 2. $\lim _{n \rightarrow \infty} \Delta_{n}=0$.

For any $n$, let $y_{n}$ be the projection of $x_{n}$ on the closed convex subset $L\left(\lambda_{n}\right)$. The sequence $\left(y_{n}\right)_{n}$ is asymptotically feasible since $d\left(y_{n}, C\right) \leqslant\left\|x_{n}-y_{n}\right\| \leqslant \Delta_{n}$.

On the other hand, an easy computation (see [3, Lemma 6.1]) shows that $f\left(y_{n}\right)=\lambda_{n}$ and that $y_{n}^{*}=\alpha_{n}\left(x_{n}-y_{n}\right) \in \partial f\left(y_{n}\right)$ for some $\alpha_{n}>0$. It follows that

$$
\left\|y_{n}^{*}\right\|=\alpha_{n}\left\|x_{n}-y_{n}\right\| \leqslant \frac{f\left(x_{n}\right)-f\left(y_{n}\right)}{\left\|x_{n}-y_{n}\right\|} \leqslant K \frac{f\left(x_{n}\right)-f\left(y_{n}\right)}{d\left(x_{n}, L\left(\lambda_{n}\right) \cap C\right)}=K \varepsilon_{n} .
$$

We conclude that $\left(y_{n}\right)_{n}$ is a stationary sequence and thus a locally minimising sequence with $\lim _{n \rightarrow \infty} f\left(y_{n}\right)=\lim _{n \rightarrow \infty} \lambda_{n}=\bar{\lambda} \in J \cap \bar{\Lambda}$ which is impossible.

Finally, combining Theorem 4.4 with Theorem 4.7, we obtain the following characterisation of constrained optimisation problem for which each stationary sequence is a locally minimising sequence:

Under the hypothesis of Theorem 4.7, and assuming moreover that any stationary sequence is feasible, then

$(f, C)$ has a constrained asymptotical well behaviour $\Longleftrightarrow(f, C) \in \mathcal{F}_{c}$.

\section{REFERENCES}

[1] P. Angleraud, 'Caractérisation duale du bon comportement de fonctions convexes', C.R. Acad. Sci. Paris Sér. I Math. 314 (1992), 583-586.

[2] A. Auslender and J.-P. Crouzeix, 'Well behaved asymptotical convex functions', Ann. Inst. H. Poincaré Anal. Non Linéaire 6 (1989), 101-122. 
[3] A. Auslender, R. Cominetti, and J.-P. Crouzeix, 'Convex functions with unbounded level sets and applications to duality theory', SIAM J. Optim. 3 (1993), 669-687.

[4] D. Aussel, J.-N. Corvellec and M. Lassonde, 'Mean value property and subdifferential criteria for lower semicontinuous functions', Trans. Amer. Math. Soc. 347 (1995), 4147-4161.

[5] D. Aussel, J.-N. Corvellec and M. Lassonde, 'Nonsmooth constrained optimization and multidirectionel mean value theorem', SIAM J. Optim. 9 (1999), 690-706.

[6] B. Bernoussi, S. Bolintineanu and C.C. Chou, 'Pareto optimizing and scalarly stationary sequences', J. Math. Anal. Appl. 220 (1998), 553-561.

[7] J.M. Borwein and D. Preiss, 'A smooth variational principle with applications to subdifferentiability and to differentiability of convex functions', Trans. Amer. Math. Soc. 303 (1987), 517-527.

[8] M. Castellani and M. Pappalardo, 'Characterizations of $\rho$-convex functions', Nonconvex Optim. Anal. 27 (1998), 219-233.

[9] F.H. Clarke, Optimization and nonsmooth analysis, Classics Appl. Math. 5, 2nd edition (SIAM, Philadelphia, PA, 1990).

[10] B. Cornet, 'Existence of slow solutions for a class of differential inclusion', J. Math. Anal. Appl. 96 (1983), 130-147.

[11] B. Cornet, 'Lipschitzian solutions of perturbed nonlinear programming problems', SIAM J. Control Optim. 24 (1986), 1123-1137.

[12] C.C. Chou, K.F. Ng and J.-S. Pang, 'Minimizing and stationary sequences of constrained optimization problems', SIAM J. Control Optim. 36 (1998), 1908-1936.

[13] R. Deville, G. Godefroy and V. Zizler, Smoothness and renormings in Banach spaces (Longman Scientific and Technical, London, 1993).

[14] I. Ekeland, 'On the variational principle', J. Math. Anal. Appl. 47 (1974), 324-353.

[15] L.R. Huang, K.-F. Ng and J.-P. Penot, 'On minimizing and critical sequences in nonsmooth optimization', SIAM J. Optim. 10 (2000), 999-1019.

[16] R. Janin, Sur la dualité et la sensibilité dans les problèmes de programmation mathématiques, (Thèse de doctorat) (Universitde Paris VI, 1974).

[17] B. Lemaire, 'Bonne position, conditionnement, et bon comportement asymptotique', Sem. Anal. Convexe 22 (1992).

[18] J.-S. Pang, 'Error bounds in mathematical programming', Math. Programming 79 (1997), 299-332.

[19] J.-P. Penot, 'Well-behavior, well-posedness and nonsmooth analysis', Pliska. Stud. Math. Bulgar. 12 (1998), 141-190.

[20] J.-P. Penot, 'Genericity of well-posedness, perturbations and smooth variational principles', Set-Valued Anal. 9 (2001), 131-157.

[21] R.R. Phelps, Convex functions, monotone operators and differentiability, Lectures Notes in Mathematics 1364, (Second edition) (Springer-Verlag, Berlin, 1993).

[22] M.J.D. Powell, 'Some global convergence properties of a variable metric algorithm for minimization without exact line searches', (Proc. Symp. Appl. Math. New York City 1975), in Nonlinear Programming (American Mathematical Society, Profidence, R.I., 1976), pp. 53-72. 
[23] R.T. Rockafellar, 'Favorable classes of Lipschitz continuous functions in subgradient optimization', in Progress in Nondifferential Optimization, IIASA Collab. Proc. Ser. (IIASA, Laxenburg, 1982), pp. 125-143.

[24] J.-P. Vial, 'Strong and weak convexity of sets and functions', Math. Oper. Res. 8 (1983), 231-259.

Laboratoire MANO

Université de Perpignan

$52 \mathrm{Av}$. de Villeneuve

66860 Perpignan Cedex

France

e-mail: aussel@univ-perp.fr

chou@univ-perp.fr 